\title{
Tumores das Glândulas Salivares
}

\author{
Salivary gland tumors
}

\section{INTRODUÇÃO}

As estatísticas mostram que $95 \%$ dos nódulos pal páveis da glândula parótida são de origem tumoral, sendo esta glândula a mais freqüentemente acometida. 0 palato é o sítio mais comum dos tumores de glândulas salivares menores. Aproximadamente 25\% dos tumores da parótida, $50 \%$ dos tumores da submandibular, $81 \%$ dos tumores das salivares menores são malignos.

0 tumor maligno mais comum da glândula Parótida é o Carcinoma M ucoepidermóide. o Carcinoma Adenóide Cístico é o tumor maligno mais freqüente da glândula Submandibular e das glândulas salivares menores.

O s tumores malignos das glândulas salivares, de baixo grau de malignidade e em estádios iniciais, são usualmente curáveis por ressecção adequada como único tratamento. Este prognóstico também é influenciado pela localização da lesão: melhor na parótida que na glândula submandibular e menos favorável na glândula sublingual ou nas glândulas salivares menores.

\section{Prognóstico}

0 prognóstico, portanto, depende da localização, do tipo histológico, do grau de diferenciação e estádio clínico do tumor:

\begin{tabular}{|c|c|c|c|}
\hline \multicolumn{2}{|c|}{ Estádio } & \multicolumn{2}{|c|}{ Sobrevida em 10 anos $(\%)$} \\
\hline \multicolumn{2}{|c|}{ Estbdiol } & \multicolumn{2}{|c|}{9054} \\
\hline \multicolumn{2}{|c|}{ Estidio II } & \multicolumn{2}{|r|}{$65 \%$} \\
\hline \multicolumn{2}{|c|}{ Estodio III//N } & \multicolumn{2}{|r|}{2236} \\
\hline \multicolumn{4}{|c|}{$\begin{array}{l}\text { Sobrevida global por localizaçāo de tumor } \\
\text { maligno }\end{array}$} \\
\hline Anos & $\begin{array}{c}\text { Parćtida } \\
\text { of }\end{array}$ & $\begin{array}{c}\text { Submantibulat } \\
5 \%\end{array}$ & $\begin{array}{l}\text { Gidndulas solinares menores } \\
\qquad \%\end{array}$ \\
\hline 5 & 55 & 31 & 48 \\
\hline 10 & 47 & 22 & 37 \\
\hline 15 & 40 & 15 & 23 \\
\hline 20 & 33 & 14 & 15 \\
\hline
\end{tabular}

\section{EXAMES DE AVALIAÇÃO}

\section{CONFIRMAÇÃO DIAGNÓSTICA}

0 diagnóstico dos tumores das glândulas salivares maiores e menores é clínico. Sinais tais como a fixação do tumor, a indefinição em relação às estruturas adjacentes, e a presença de paralisia facial são fatores indicativos de malignidade. $D$ entre os exames de rotina utilizados para se avaliar as lesões de glândulas salivares maiores, não se pode prescindir da punção aspirativa com agulha fina para diagnóstico cito-patológico. Para diagnóstico dos tumores das glândulas salivares menores pode ser necessário a punção com agulhafina, ou biópsia incisional. É importante ressaltar que a biópsia incisional só deve ser realizada quando o diagnóstico histopatológico irá definir a extensão da cirurgia.

\section{Avaliação da EXTENSÃO da dOENÇA}

O s critérios de extensão dos tumores das glândulas salivares são descritos de acordo com o tamanho, mobilidade e, no caso dos tumores parotídeos, comprometimento do nervo facial. 0 utras informações podem ser obtidas através de exames mais complexos, como por exemplo, a tomografia computadorizada.

\section{EXAMES DE EXCEÇÃO}

- Radiografia simples de mandíbula (para avaliação de invasão mandibular pelos tumores de glândula submandibular ou parótida);

- Tomografia computadorizada (para avaliação de invasão das estruturas profundas e tumores do lobo profundo da parótida);

- Radiografia simples dos seios da face (em tumores de palato);

- Estudo radiológico de osso temporal (tumores malignos da parótida);

- Ultra-sonografia para diagnóstico diferencial entre linfonodo cervical, processos inflamatórios, cálculos e tumores; 
- Ressonância magnética (exclusivamente quando a extensão da lesão for de difícil determinação clínica), principalmente nos tumores de lobo profundo da parótida.

\section{TRATAMENTO}

\section{TUMORES BENIGNOS}

\section{Parótida}

- Tumor do lobo superficial: parotidectomia superficial parcial ou completa, com conservação do nervo facial;

- Tumor do lobo profundo: parotidectomia total com conservação do nervo facial.

\section{Submandibular \\ - Submandibulectomia.}

\section{Outros}

- Ressecção da lesão com margem de segurança podendo incluir partes ósseas, nos casos de tumores no palato.

\section{TUMORES MALIGNOS - TRATAMENTO POR ESTÁDIOS*}

\section{Parótida}

\section{Estádio I e II}

$\mathrm{N}$ eoplasias de baixo grau ou alto grau de malignidade. 0 tratamento será sempre cirúrgico, buscando sempre preservar o nervo facial.

A radioterapia complementar será sempre indicada em casos de tumores de alto grau de malignidade, ou em casos de margens cirúrgicas insuficientes ou em tumores de difícil acesso (lobo profundo).

A radioterapia exclusiva é indicação de exceção ou como tratamento paliativo, em casos considerados sem indicação para a cirurgia.

\section{Estádio III}

$\mathrm{N}$ eoplasias de baixo grau ou alto grau de malignidade. 0 tratamento de escolha é sempre o cirúrgico. 0 nervo facial deverá ser preservado quando possível. (Ausência de invasão macroscópica)
0 esvaziamento cervical deve ser associado em casos de invasão cervical por tumores de alto grau de malignidade ou em tumores que apresentem um alto índice de metástase cervical (Carcinoma Epidermóide, Carcinoma Indiferenciado).

0 volume de tratamento radioterápico inclui o leito operatório com $2 \mathrm{~cm}$ de margem, região nodal subdigástrica ipsilateral, em caso de Carcinoma adenóide cístico, sendo que todo 0 trajeto do nervo facial é incluído no volume de tratamento. Lesões profundas são abordadas com distribuição de campos laterais, com filtros compensadores para melhoria de isodose, ou feixe de elétrons de 12-17 Mev de forma isolada ou em combinação com feixe de fótons. A dose prescrita é de 55-60 Gy diárias de 1.8 a $2 \mathrm{G} \mathrm{y.}$ O s linfonodos cervicais ipsilaterais são irradiados, em caso de doença residual e tumores de alto grau (com exceção de carcinoma adenóide cístico pelo baixo risco de recidiva cervical) com dose prescrita é de 50 Gy em 25 sessões.

\section{Estádio IV}

O s tumores avançados das glândulas salivares deverão ser tratados de acordo com a avaliação de operabilidade do tumor e do paciente.

$\mathrm{N}$ os casos em que existe possibilidade clínica para o paciente se submeter ao tratamento cirúrgico, este deverá ser realizado sempre com a indicação de radioterapia complementar.

\section{Submandibular \\ Estádio I e II}

$\mathrm{N}$ eoplasias de baixo grau de malignidade - Submandibulectomia e esvaziamento da região submandibular.

$\mathrm{N}$ eoplasias de alto grau de malignidade Esvaziamento Cervical Supraomohióideo + radioterapia. O s critérios para indicação e aplicação de radioterapia pós-operatória são os mesmos mencionados para os tumores da parótida.

\section{Estádio III}

$\mathrm{N}$ eoplasias debai xo ealto grau demalignidade - Esvaziamento Supraomohióideo + radioterapia. O s critérios para indicação e aplicação de

* M inistério da Saúde (Brasil). TN M Classificação de tumores malignos. [trad. 5a ed. UICC, 1997]. Rio de Janeiro; 1998. 
radioterapia pós-operatória são os mesmos mencionados para os tumores da parótida.

\section{Estádio IV}

O stumoresavançados dasglândulassalivares deverão ser tratados de acordo com a avaliação de operabilidade do tumor e do paciente.

$\mathrm{N}$ os casos em que existe possibilidade clínica para o paciente se submeter ao tratamento cirúrgico, este deverá ser realizado sempre com a indicação de radioterapia complementar.

A radioterapia préoperatória é indicação de exceção, pois ela deve ser feita como única arma terapêutica para os tumores neste estádio. 0 s critérios para indicação eaplicação de radioterapia pós-operatória são os mesmos mencionados para tumores da parótida.

\section{GLÂNDULAS SALIVARES MENORES}

O s tumores das glândulas salivares menores terão sempre indicação de tratamento cirúrgico, que será a ressecção alargada da lesão, e radioterapia complementar nos casos em que houver margem cirúrgica acometida ou nos tumores de alto grau de malignidade. 0 volume de tratamento radioterápico vai depender do epicentro do tumor, e segue os mesmos princípios do tratamento dos tumores epiteliais desta área, com exceção do carcinoma adenóide cístico, que requer, irradiação de seguimentos nervosos ate a base do crânio. As doses prescritas são: $60 \mathrm{G}$ y para irradiação profilática, 66 Gy em caso de doença residual microscópica, 70 Gy para doença residual macroscópica, em frações de 1.8 a 2 Gy.

\section{TUMOR RECIDIVADO}

O s tumores recidivados das glândulas salivares serão tratados conforme o tipo histológico, tratamento prévio, sítio da recidiva eextensão tumoral e, "status" clínico do paciente.

\section{EXAMES DE SEGUIMENTO}

O s exames clínicos de seguimento incluem o exame da cavidade oral além da palpação da região parotídea ou submandibular, edo pescoço.
A realização anual de raios $X$ de Tórax deverá ser efetuada no seguimento detumores malignos de al to grau de malignidade.

A laringoscopia deverá ser efetuada em casos de queixas relativas a laringe ou hipofaringe.

0 seguimento deveráser feito bimestralmente no primeiro ano; quadrimensalmenteno segundo ano; semestralmente no terceiro ano; e anualmente a partir do quarto ano.

Os doentes tratados de tumores benignos e de baixo grau de malignidade podem, depois de um ano, receber alta e serem orientados para retorno.

\section{NOTA FINAL}

Para o IN CA, qualquer conduta aplicada em suas unidades hospitalares que se encontre fora das aqui especificadas é considerada experimental ou irregular.

\section{BIBLIOGRAFIA}

1. Armstrong J G, H arrison LB, Spiro RH, Fass $D E$, Strong EW, FuksZY. O bservations on the natural history and treatment of recurrent major salivary gland cancer. J Surg 0 ncol 1990;44:138-41.

2. ArmstrongJ $G, H$ arrison $L B, T$ haler $H T$, Friedlander-K lar H , Fass D E, Z elefsky M J, Shah JP, Strong EW, Spiro RH . Theindications for electivetreatment of theneck in cancer of themajor sal ivary glands. C ancer 1992;69:615-9.

3. Batsakis JG. Staging of salivary gland neoplasms: role of histopathologic and molecular factors. Am J Surg 1994;168:386-90.

4. Beckhardt RN, Weber RS, ZaneR, Garden AS, Wolf $P, C$ arrillo R, Luna M A. M inor salivary gland tumors of the palate: clinical and pathologic correlates of outcome. Laryngoscope 1995;105:1155-60.

5. Garden AS, Weber RS, AngKK, M orrison WH, $M$ atre J, Peters LJ. Postoperative radiation therapy for malignant tumors of minor salivary glands. C ancer 1994;73:2563-9. 
6. H anna D C, D ickason W L, Richardson GS, G aisford JC. M anagement of recurrent salivary gland tumors. Am J Surg 1976;132:453-8.

7. HicksM J, el-N aggar AK, ByersRM , FlaitzCM, Luna M A, Batsakis JG. Prognostic factors in mucoepidermoid carcinomas of major salivary glands: a clinicopathologic and flow cytometric study. Eur J Cancer B Oral Oncol 1998;30b(5):329-34.

8. KlineTS, M errian JM , Shapshay SM . Aspiration biopsy cytology of the salivary gland. AJCP 1981;76(3):263-9.

9. LimaRA, FreitasEQ, DiasFL, deSarvat MA, H olguin E, deSáG M, Kligerman J. Tumor de Warthin: estudo retrospectivo de 45 casos. Rev Col Bras Cir 1991;18:236-9.

10. Lima RA, Kligerman J. Glândulas salivares. In: VinháesJC. Clínicaeterapêuticacirúrgicas. Rio deJaneiro: GunabaraKoogan; 1997. p. 336-41.

11. M edinaJ E. N eck dissection in thetreatment of cancer of themajor salivary glands. 0 tolaryngol Clin N orth Am 1998;31:815-22.

12. $N$ ascimento AG, Amaral ALP, Prado LAF, Kligerman J, SilveiraT RP. Adenoid cystic carcinoma of salivary glands: a study of 61 cases with clinicopathologic correlation. Cancer 1986;57:312-9.

13. N ascimento AG, Amaral ALP, Prado LAF, Kligerman J, SilveiraT R P. M ucoepidermoid car- cinoma of salivary glands: a clinicopathologic study of 46 cases. H ead N eck 1986;8:409-17.

14. Ö stman J, Anneroth G, Gustafsson H , Tavelin B. M alignant salivary gland tumours in Sweden 1960-1989: an epidemiological study. Eur J Cancer B O ral O ncol 1997;33:169-76.

15. Pitts D B, H ilsinger RL Jr, Karandy E, RossJC, CaroJE. Fine-needleaspiration in the diagnosis of salivary gland disorders in the community hospital setting. Arch $\mathrm{O}$ tolaryngol $\mathrm{H}$ ead N eck Surg 1992;118:479-82.

16. Shah JP, Ihde JK. Salivary gland tumors. Curr Probl Surg 1990;27:775-883.

17. Spiro RH . Salivary N eoplasms: overview of a 35-year experiencewith 2807 patients. $\mathrm{H}$ ead N eck 1986;8:177-84.

18. Spiro RH . Changing trendsin themanagement of salivary tumors. Semin Surg O ncol 1995; 11:240-5.

19. Spiro RH . M anagement of malignant tumors of thesalivary glands. O ncology 1998;12:671-83.

20. Spiro RH , ArmstrongJ G , H arrison LB, G eller $N$ L, Lin SY, Strong EW. Carcinoma of major salivary glands-recent trends. Arch 0 tolaryngol $\mathrm{H}$ ead N eck Surg 1989;115:316-21.

21. Tran L, Sadeghi A, $H$ anson D, Juillard $G, M$ ackintosh R, C alcaterraTC, Parker RG. M ajor salivary gland tumor: treatment results and prognostic factors. Laryngoscope 1986;96:1139-44. 